\title{
Fixed Point and Common Fixed Point Theorems for Cyclic Quasi-Contractions in Metric and Ultrametric Spaces
}

\author{
Parin Chaipunya ${ }^{1}$, Yeol Je Cho ${ }^{2 *}$, Wutiphol Sintunavarat ${ }^{1}$, Poom Kumam ${ }^{1 *}$ \\ ${ }^{1}$ Department of Mathematics, Faculty of Science, King Mongkut's University of \\ Technology Thonburi, Bangkok, Thailand \\ ${ }^{2}$ Department of Mathematics Education and the RINS, Gyeongsang National University, Chinju, Korea \\ Email: chaipunya.p@gmail.com, ${ }^{*}$ yjcho@gnu.ac.kr, poom_teun@hotmail.com, ${ }^{*}$ poom.kum@kmutt.ac.th
}

Received August 10, 2012; revised September 25, 2012; accepted October 5, 2012

\begin{abstract}
In this paper, we prove introduce some fixed point theorems for quasi-contraction under the cyclical conditions. Then, we point out that a common fixed point extension is also applicable via our earlier results equipped together with a weaker cyclical properties, namely a co-cyclic representation. Examples are as well provided along this paper.
\end{abstract}

Keywords: Cyclic Quasi-Contraction; Co-Cyclic Quasi-Contraction; Metric Space; Ultrametric Space

\section{Introduction}

Since Banach [1] proved his contraction principle in 1922, many authors have improved, extended and generalized Banach's contraction principle in several ways and applied the principle to differential and integral equations, variational inequality theory, complementarity problems, equilibrium problems, optimization problems, convex analysis and many others.

Theorem 1.1 [1] Let $f$ be a self-mapping on a complete metric space $(X, d)$. If there exists $k \in[0,1)$ such that

$$
d(f x, f y) \leq k d(x, y)
$$

for all $x, y \in X$, then $f$ has a unique fixed point in $X$.

In 1971, Ććirić [2] generalized Banach's contraction principle theorem to a more general contraction as follows:

Theorem 1.2 [2] Let $(X, d)$ be a complete metric space and $f: X \rightarrow X$ be a mapping such that, for all $x, y \in X$, there exists $\alpha, \beta, \gamma, \delta \geq 0$ with $\alpha+\beta+\gamma+2 \delta<1$ such that

$$
\begin{aligned}
d(f x, f y) \leq & \alpha d(x, y)+\beta d(x, f x)+\gamma d(y, f y) \\
& +\delta[d(x, f y)+d(y, f x)] .
\end{aligned}
$$

Then $f$ has a unique fixed point.

Also, in 1974, Ććirić [3] generalized his own result [2] by introducing the quasi-contraction and proved a fixed point theorem under this condition as follows:

Definition 1.3 [3] A mapping $f$ of a metric space $(X, d)$ into itself is said to be a quasi-contraction if

\footnotetext{
${ }^{*}$ Corresponding authors.
}

there exists a number $0 \leq \alpha<1$ such that

$$
\begin{gathered}
d(f x, f y) \leq \alpha \max \{d(x, y), d(x, f x), d(y, f y), \\
d(x, f y), d(y, f x)\}
\end{gathered}
$$

for all $x, y \in X$.

Theorem 1.4. [3] Let $(X, d)$ be a complete metric space and $f: X \rightarrow X$ be a quasi-contraction. Then $f$ has a unique fixed point in $X$.

In 2005, Rus [4] introduced the cyclical condition in metric spaces. For some results on fixed point theory, we refer the readers to [5-7]. Throughout this paper, we denote the set $\{m \in \mathbb{N} \bigcup\{0\}: m \leq n$ for some $n \in \mathbb{N}\}$ by $\mathbb{N}_{0}^{n}$.

Definition 1.5. [4] Let $X$ be a nonempty set and $f: X \rightarrow X$ be a mapping. The set $\bigcup_{i \in \mathbb{N}_{0}^{m-1}} X_{i}$ is called a cyclic representation of $X$ with respect to $f$ if the following conditions hold:

1) $X=\bigcup_{i \in \mathbb{N}_{0}^{m-1}} X_{i}$

2) $X_{i}$ is a nonempty subset of $X$ for all $i \in \mathbb{N}_{0}^{m-1}$;

3) $f\left(X_{i}\right) \subseteq X_{i+1(\bmod m)}$ for all $i \in \mathbb{N}_{0}^{m-1}$.

Definition 1.6. Let $X=[0,2], X_{0}=[0,1]$,

$X_{1}=\left[\frac{1}{2}, \frac{3}{2}\right]$ and $X_{2}=[1,2]$. Now, we define a selfmapping $f$ on $X$ by

$$
f(x)=\left\{\begin{array}{lll}
x+\frac{1}{2} & \text { if } & 0 \leq x \leq \frac{1}{2} \\
1 & \text { if } & \frac{1}{2}<x \leq \frac{3}{2} \\
x-1 & \text { if } & \frac{3}{2}<x \leq 2 .
\end{array}\right.
$$


Now, we see that $f\left(X_{0}\right)=\left[\frac{1}{2}, 1\right] \subseteq\left[\frac{1}{2}, \frac{3}{2}\right]=X_{1}$,

$f\left(X_{1}\right)=\{1\} \subseteq[1,2]=X_{2}$ and

$f\left(X_{2}\right)=\left(\frac{1}{2}, 1\right] \subseteq[0,1]=X_{0}$. Therefore, the set $\bigcup_{i \in \mathbb{N}_{0}^{2}} A_{i}$

is a cyclic representation of $X$ with respect to $f$.

Definition 1.7. Let $X$ be a nonempty set, $f: X \rightarrow X$ be a mapping and the set $\bigcup_{i \in \mathbb{N}_{0}^{m-1}} X_{i}$ be a cyclic representation of $X$ with respect to $f$. If $x_{1} \in X_{i}$ and $x^{2} \subseteq X_{i+1(\bmod m)}$ for some $i \in \mathbb{N}_{0}^{m-1}$, we say that $x_{1}$ and $x_{2}$ are descendants in $Y$.

The purpose of this paper is to extend Ććirić's quasicontraction to a cyclic quasi-contraction, establish some fixed point theorems and give an example to illustrate the main result. We also introduce the notion of co-cyclic quasi-contraction and prove some common fixed point theorems.

\section{Fixed Point Theorems}

In this section, we introduce a generalization of Ććirić's quasi-contraction, say, a cyclic quasi-contraction, and prove some fixed point theorems.

Definition 2.1. Let $A_{0}, A_{1}, \cdots, A_{m-1}$ be nonempty closed subsets of a metric space $(X, d), Y:=\bigcup_{i \in \mathbb{N}_{0}^{m-1}} A_{i}$ and $f: Y \rightarrow Y$ be a mapping. If the following conditions are satisfied:

1) $\bigcup_{i \in \mathbb{N}_{0}^{m-1}} A_{i}$ is a cyclic representation of $Y$ with respect to $f$

2) there exists $0 \leq \alpha<1$ such that

$$
\begin{gathered}
d(f x, f y) \leq \alpha \max \{d(x, y), d(x, f x), d(y, f y), \\
d(x, f y), d(y, f x)\}
\end{gathered}
$$

whenever $x$ and $y$ are descendants in $Y$, then $f$ is called a cyclic quasi-contraction.

Remark 2.2. To reduce a cyclic quasi-contraction to a quasi-contraction, simply take each $A_{i}=X$ and the result directly emerges.

Now, we can construct some fixed point theorems, which generalize the further results, as follows:

Theorem 2.3. Let $A_{0}, A_{1}, \cdots, A_{m-1}$ be nonempty closed subsets of a complete metric space $(X, d)$ and $Y:=\bigcup_{i \in \mathbb{N}_{0}^{m-1}} A_{i}$. Suppose that $f: Y \rightarrow Y$ is a cyclic quasi-contraction with $\alpha \in\left[0, \frac{1}{2}\right)$. Then $f$ has a unique fixed point $z^{*} \in \bigcap_{i \in \mathbb{N}_{0}^{m-1}} A_{i}$ and the sequence $\left\{f^{n} x_{0}\right\}_{n=1}^{\infty}$ converges to $z^{*}$ for any $x_{0} \in Y$

Notice that the $\alpha$-constant in the quasi-contraction is restricted to the set $\left[0, \frac{1}{2}\right)$. Next, we can drop this restriction and develop a theorem in an ultrametric space. The result follows from the additional assumption of an ultrametric space. Before we give the result, we now give the definition of an ultrametric space.

Definition 2.4. Let $X$ be a nonempty set. A function $\alpha \in d: X \times X \rightarrow \mathbb{R}$ is called an ultrametric if it satisfies the following conditions:

1) $d(x, y) \geq 0$ and $d(x, y)=0$ if and only if $x=y$ for all $x, y \in X$;

2) $d(x, y)=d(y, x)$ for all $x, y \in X$;

3) $d(x, y) \leq \max \{d(x, z), d(z, y)\}$ for all $x, y, z \in X$.

A set $X$ equipped with this ultrametric $d$, denoted $(X, d)$, is called an ultrametric space.

Remark 2.5. Note that an ultrametric space is also a metric space. We can simply prove this. In fact, for any $x, y, z \in X$,

$$
\begin{aligned}
& d(x, y) \leq \max \{d(x, z), d(z, y)\} \\
& \leq \max \{d(x, z), d(z, y)\}+\min \{d(x, z), d(z, y)\} \\
& =d(x, z), d(z, y),
\end{aligned}
$$

which in turn is a metric.

Theorem 2.6. Let $A_{0}, A_{1}, \cdots, A_{m-1}$ be nonempty closed subsets of a complete ultrametric space $(X, d)$ and $Y:=\bigcup_{i \in \mathbb{N}_{0}^{m-1}} A_{i}$. Suppose that $f: Y \rightarrow Y$ is a cyclic quasi-contraction. Then $f$ has a unique fixed point

$z^{*} \in \bigcap_{i \in \mathbb{N}_{0}^{m-1}} A_{i}$ and the sequence $\left\{f^{n} x_{0}\right\}_{n=1}^{+\infty}$ converges to $z^{*}$ for any $x_{0} \in Y$.

Now, we prove Theorem 2.3. The proof of Theorem 2.6 is quite similar to the proof of Theorem 2.3, we omit to prove this theorem.

Proof of Theorem 2.3. Let $x_{0} \in Y$ be arbitrarily chosen. Define a sequence $\left\{x_{n}\right\}_{n=1}^{\infty}$ by $x_{n}=f^{n} x_{0}$ for all $n \geq 1$. If there exists a positive integer $n_{0}$ such that $x_{n_{0}}=f x_{n_{0}}$, the the proof is finished. So, assume that $x_{n-1} \neq x_{n}$ for all $n \geq 1$. Since $x_{0} \in Y$, there exists $i_{0} \in \mathbb{N}_{0}^{m-1}$ such that $x_{0} \in A_{i_{0}}$. Therefore, $x_{1}=f x_{0} \in A_{i_{0}+1(\bmod m)}$ and, by induction, we have $x_{n}=f^{n} x_{0} \in A_{i_{0}+n(\bmod m)}$. Hence we have

$$
\begin{aligned}
d & \left.d x_{n}, x_{n+1}\right)=d\left(f x_{n-1}, f x_{n}\right) \\
\leq & \alpha \max \left\{d\left(x_{n-1}, x_{n}\right), d\left(x_{n-1}, x_{n}\right), d\left(x_{n}, x_{n+1}\right),\right. \\
& \left.d\left(x_{n-1}, x_{n+1}\right), d\left(x_{n}, x_{n}\right)\right\} \\
= & \alpha \max \left\{d\left(x_{n-1}, x_{n}\right), d\left(x_{n}, x_{n+1}\right), d\left(x_{n-1}, x_{n+1}\right)\right\}
\end{aligned}
$$

Assume that 


$$
\begin{aligned}
& \max \left\{d\left(x_{n-1}, x_{n}\right), d\left(x_{n}, x_{n+1}\right), d\left(x_{n-1}, x_{n+1}\right)\right\} \\
& =d\left(x_{n}, x_{n+1}\right) .
\end{aligned}
$$

Then we can see that $d\left(x_{n-1}, x_{n}\right) \leq \alpha d\left(x_{n}, x_{n+1}\right)$, which is a contradiction. Therefore, from (1), it follows that

$$
\begin{aligned}
& d\left(x_{n}, x_{n+1}\right) \leq \alpha \max \left\{d\left(x_{n-1}, x_{n}\right), d\left(x_{n-1}, x_{n+1}\right)\right\} \\
& \leq \alpha \max \left\{d\left(x_{n-1}, x_{n}\right), d\left(x_{n-1}, x_{n}\right)+d\left(x_{n}, x_{n+1}\right)\right\} \\
& =\alpha\left[d\left(x_{n-1}, x_{n}\right)+d\left(x_{n}, x_{n+1}\right)\right] .
\end{aligned}
$$

Consequently, we can deduce that

$d\left(x_{n}, x_{n+1}\right) \leq \lambda d\left(x_{n-1}, x_{n}\right)$, where $\lambda=\frac{\alpha}{1-\alpha}<1$. By repeating this process, we have $d\left(x_{n}, x_{n+1}\right) \leq \lambda^{n} d\left(x_{0}, x_{1}\right)$. Thus it is easily seen that $\left\{x_{n}\right\}_{n=1}^{\infty}$ is a Cauchy sequence in $Y$. Since $Y$ is closed in $X, Y$ is a complete subspace of $X$ and so $\left\{x_{n}\right\}_{n=1}^{\infty}$ converges to some point $x^{*} \in Y$. Denote $Z \in \bigcap_{i \in \mathbb{N}_{0}^{m-1}} A_{i}$. Now, we show that $x^{*} \in Z$. Since, for each $n \geq 1$, we have $x_{n} \in A_{i_{0}+n(\bmod m)}$, it is easy to see that, for each $i \in \mathbb{N}_{0}^{m-1}, A_{i}$ contains infinitely many points of $\left\{x_{n}\right\}_{n=1}^{\infty}$. Since $A_{i}$ is closed for each $i \in \mathbb{N}_{0}^{m-1}$, we can construct a subsequence of $\left\{x_{n}\right\}_{n=1}^{\infty}$ in $A_{i}$ which converges to $x^{*}$. Therefore, $x^{*} \in Z$. In other words, $Z$ is nonempty.

Consider the restriction $\left.f\right|_{z}$ of the function $f$. We can see that it maps $Z$ into itself, i.e., $\left.f\right|_{Z}: Z \rightarrow Z$. Then it is easily proved, by applying Theorem 1.4 , that $\left.f\right|_{Z}$ has a unique fixed point $x^{*} \in Z$. Thus $Z^{*}$ is also a fixed point of $f$.

Now, assume that there exists another fixed point of $f$ denoted by $\bar{x} \in Y$. Since $\bar{x} \in Y$, we have $\bar{x} \in A_{j}$ for some $j \in \mathbb{N}_{0}^{m-1}$. Therefore, $\bar{x}$ and $z^{*}$ are descendants in $Y$. Hence we have

$$
\begin{aligned}
& d\left(\bar{x}, z^{*}\right)=d\left(f \bar{x}, f z^{*}\right) \\
\leq & \alpha \max \left\{d\left(\bar{x}, z^{*}\right), d(\bar{x}, f \bar{x}), d\left(z^{*}, f z^{*}\right),\right. \\
& \left.d\left(f \bar{x}, f z^{*}\right), d\left(z^{*}, f \bar{x}\right)\right\} \\
= & \alpha d\left(\bar{x}, z^{*}\right),
\end{aligned}
$$

which implies that $d\left(\bar{x}, z^{*}\right)=0$, that is, $z^{*}$ is the unique fixed point of $f$.

Next, we show that $x^{*}=z^{*}$. Since $Z$ and $z^{*} \in A_{i_{0}+n+1(\bmod m)}$, we have

$$
\begin{aligned}
d & \left.d x_{n}, z^{*}\right)=d\left(f x_{n-1}, f z^{*}\right) \\
\leq & \alpha \max \left\{d\left(x_{n-1}, z^{*}\right), d\left(x_{n-1}, x_{n}\right), d\left(z^{*}, f z^{*}\right),\right. \\
& \left.d\left(x_{n-1}, f z^{*}\right), d\left(x_{n}, z^{*}\right)\right\} \\
\leq & \alpha \max \left\{d\left(x_{n-1}, z^{*}\right), d\left(x_{n-1}, x_{n}\right), d\left(x_{n}, z^{*}\right)\right\} .
\end{aligned}
$$

We have a contradiction if

$$
\max \left\{d\left(x_{n-1}, z^{*}\right), d\left(x_{n-1}, x_{n}\right), d\left(x_{n}, z^{*}\right)\right\}=d\left(x_{n}, z^{*}\right) .
$$

So, we claim that

$$
\begin{aligned}
& \max \left\{d\left(x_{n-1}, z^{*}\right), d\left(x_{n-1}, x_{n}\right), d\left(x_{n}, z^{*}\right)\right\} \\
& =\max \left\{d\left(x_{n-1}, z^{*}\right), d\left(x_{n-1}, x_{n}\right)\right\} .
\end{aligned}
$$

Hence, from (2), it follows that

$$
\begin{aligned}
d\left(x_{n}, z^{*}\right) & \leq \alpha \max \left\{d\left(x_{n-1}, z^{*}\right), d\left(x_{n-1}, x_{n}\right)\right\} \\
& \leq \alpha \max \left\{d\left(x_{n-1}, z^{*}\right), d\left(x_{n-1}, z^{*}\right), d\left(x_{n}, z^{*}\right)\right\} \\
& =\alpha\left[d\left(x_{n-1}, z^{*}\right)+d\left(x_{n-1}, z^{*}\right)\right] \\
& \leq \lambda d\left(x_{n-1}, z^{*}\right) .
\end{aligned}
$$

Thus, by repeating this process, we obtain $d\left(x_{n}, z^{*}\right) \leq \lambda^{n} d\left(x_{0}, z^{*}\right)$. Therefore, we have $x^{*}=z^{*}$, that is, $\left\{x_{n}\right\}_{n=1}^{\infty}$ converges to the unique fixed point $z^{*}$ of $f$ in $Z$ for any initial $x_{0} \in Y$. This complete the proof.

Proof of Theorem 2.6. Let $x_{0} \in X$ be arbitrary. Define a sequence $\left\{x_{n}\right\}_{n=1}^{+\infty}$ as in the proof of Theorem 2.3.

Following the proof lines, we obtain

$$
\begin{aligned}
& d\left\{x_{n}, x_{n+1}\right\} \leq \alpha \max \left\{d\left(x_{n-1}, x_{n}\right), d\left(x_{n-1}, x_{n+1}\right)\right\} \\
& \leq \alpha \max \left\{d\left(x_{n-1}, x_{n}\right), \max \left\{d\left(x_{n-1}, x_{n}\right), d\left(x_{n}, x_{n+1}\right)\right\}\right\} \\
& =\alpha d\left(x_{n-1}, x_{n}\right) .
\end{aligned}
$$

By repeating this process, we get $d\left(x_{n-1}, x_{n}\right) \leq \alpha^{n} d\left(x_{0}, x_{1}\right)$. Then it is easily seen that the sequence $\left\{x_{n}\right\}_{n=1}^{+\infty}$ is a Cauchy sequence and so, from the completeness of $Y,\left\{x_{n}\right\}_{n=1}^{\infty}$ converges to some point $x^{*} \in Y$. We can show, by using the proof of Theorem 2.3, that $Z \in \bigcap_{i \in \mathbb{N}_{0}^{m-1}} A_{i}$ is not empty. More precisely, $x^{*} \in Z$.

Now, we consider the restriction $\left.f\right|_{Z}: Z \rightarrow Z$ of the function $f$. Note that the strong triangle inequality also implies the ordinary triangle inequality. Hence Theorem 1.4 can be applied to confirm the existence of a unique fixed point $z^{*}$ of $\left.f\right|_{z}$ in $Z$. By the proof of Theorem 2.3, we can show that $z^{*}$ is also the unique fixed point of $f$.

Now, we show that the sequence $\left\{f^{n} x_{0}\right\}_{n=1}^{\infty}$ converges to $z^{*}$ for any $x_{0} \in Y$. Since $x_{n} \in A_{1_{0}+n(\bmod m)}$ and $z^{*} \in A_{i_{0}+n+1(\bmod m)}$, we have

$$
\begin{aligned}
& d\left(x_{n}, z^{*}\right)=d\left(f x_{n-1}, f z^{*}\right) \\
& \leq \alpha \max \left\{d\left(x_{n-1}, z^{*}\right), d\left(x_{n-1}, x_{n}\right), d\left(z^{*}, f z^{*}\right),\right.
\end{aligned}
$$




$$
\begin{aligned}
& \left.d\left(f x_{n-1}, f z^{*}\right), d\left(x_{n}, z^{*}\right)\right\} \\
& =\alpha \max \left\{d\left(x_{n-1}, z^{*}\right), d\left(x_{n-1}, x_{n}\right), d\left(x_{n}, z^{*}\right)\right\} .
\end{aligned}
$$

We can see that

$$
\max \left\{d\left(x_{n-1}, z^{*}\right), d\left(x_{n-1}, x_{n}\right), d\left(x_{n}, z^{*}\right)\right\} \neq d\left(x_{n}, z^{*}\right) .
$$

Otherwise, we have a contradiction. Hence we have

$$
\begin{aligned}
& d\left(x_{n}, z^{*}\right) \leq \alpha \max \left\{d\left(x_{n-1}, z^{*}\right), d\left(x_{n-1}, x_{n}\right)\right\} \\
& \leq \alpha \max \left\{d\left(x_{n-1}, z^{*}\right), \max \left\{d\left(x_{n-1}, z^{*}\right), d\left(x_{n}, z^{*}\right)\right\}\right\} \\
& =\alpha d\left(x_{n-1}, z^{*}\right) .
\end{aligned}
$$

Again, by repeating this procedure, we obtain $d\left(x_{n}, z^{*}\right) \leq \alpha^{n} d\left(x_{0}, z^{*}\right)$. Then the sequence $\left\{x_{n}\right\}_{n=1}^{\infty}$ converges to the unique fixed point $z^{*}$ of $f$ in $Z$ for any initial $x_{0} \in Y$. This completes the proof.

Notice that our results do not only generalize Ććirić's result, but also make it easier to determine the fixed point of a given mapping as in the following example:

Example 2.7. Consider a weighted graph $G:=\{V(G):=\{u, v, x, y\}, E(G)\}$ whose $E(G)$ makes $G$ a complete $K_{4}$ graph with weights $w_{i, j}$ for each $(i, j) \in E(G)$ given as follows:

\begin{tabular}{ll}
\hline$(i, j)$ & $w_{i, j}$ \\
\hline$(u, v)$ & 3 \\
$(u, x)$ & 1 \\
$(u, y)$ & 2 \\
$(v, x)$ & 2 \\
$(v, y)$ & 1 \\
$(x, y)$ & 3 \\
\hline
\end{tabular}

For the understanding of the readers, we illustrate $G$ as a figure in the following:

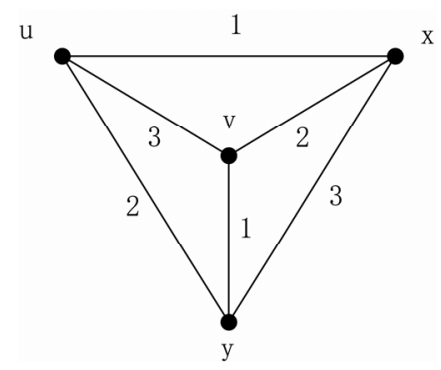

Now, define a function $d: V(G) \times V(G) \rightarrow \mathbb{R}$ by letting, for all $i, j \in V(G), d(i, j)=0$ if $i=j$ and $d(i, j)=w_{i, j}$ if $i \neq j$ (we can do this because the graph $G$ is complete). By this setting, it is easy to verify that $(V(G), d)$ is a complete metric space. Set $A_{0}:=\{u, v, x\}$ and $A_{1}:=\{v, y\}$, we have $A_{0}$ and $A_{1}$ being two closed subsets of $V(G)$. Suppose that the mapping $f: V(G) \rightarrow V(G)$ given by the following:

\begin{tabular}{cc}
\hline$i$ & $f(i)$ \\
\hline$u$ & $y$ \\
$v$ & $v$ \\
$x$ & $y$ \\
$y$ & $v$ \\
\hline
\end{tabular}

By a careful calculation, we may obtain that $f$ is a cyclic quasi-contraction on $V(G)=A_{0} \cup A_{1}$. Thus, $f$ has a unique fixed point $v \in A_{0} \cap A_{1}$ and $f^{n}(i) \rightarrow v$ for every $i \in V(G)$.

\section{Common Fixed Point Theorems}

In this section, we prove some common fixed points theorems for the co-cyclic conditions. Before we can prove our results, we also need the following, which is an extension of Definition 2 .

Definition 3.1. Let $X$ be a nonempty set and $f, g: X \rightarrow X$ be two mappings. The set $\bigcup_{i \in \mathbb{N}_{0}^{m-1}} X_{i}$ is called a co-cyclic representation of $X$ between $f$ and $g$ if the following conditions are satisfied:

1) $X=\bigcup_{i \in \mathbb{N}_{0}^{m-1}} X$;

2) $X_{i}$ is a non-empty subset of $X$ for all $i \in \mathbb{N}_{0}^{m-1}$;

3) $f\left(X_{i}\right) \subseteq g\left(X_{i+1(\bmod m)}\right)$ for all $i \in \mathbb{N}_{0}^{m-1}$.

Example 3.2. Let $X=[0,2], X_{0}=[0,1], X_{1}=\left[\frac{1}{2}, \frac{3}{2}\right]$ and $X_{2}=[1,2]$. Now, define two self-mappings $f, g$ on $X$ by

$$
\begin{gathered}
f x=\left\{\begin{array}{lll}
1 & \text { if } & 0 \leq x \leq 1, \\
x-\frac{1}{2} & \text { if } & 1<x \leq \frac{3}{2} \\
x-1 & \text { if } & \frac{3}{2}<x \leq 2,
\end{array}\right. \\
g x=\left\{\begin{array}{lll}
x+\frac{1}{2} & \text { if } & 0 \leq x \leq \frac{1}{2} \\
1 & \text { if } & \frac{1}{2}<x \leq \frac{3}{2}
\end{array}\right. \\
x-1 \quad \text { if } \quad \frac{3}{2}<x \leq 2 .
\end{gathered}
$$

Now, we see that

$$
g\left(X_{0}\right)=\left[\frac{1}{2}, 1\right], g\left(X_{1}\right)=\{1\}, g\left(X_{2}\right)=\left(\frac{1}{2}, 1\right],
$$




$$
f\left(X_{0}\right)=\{1\}, f\left(X_{1}\right)=\left(\frac{1}{2}, 1\right], f\left(X_{2}\right)=\left(\frac{1}{2}, 1\right] .
$$

Therefore, $f\left(X_{0}\right) \subseteq g\left(X_{1}\right), \quad f\left(X_{1}\right) \subseteq g\left(X_{2}\right)$ and $f\left(X_{2}\right) \subseteq g\left(X_{0}\right)$. That is, $\bigcup_{i \in \mathbb{N}_{0}} X_{i}$ is a co-cyclic representation of $X$ between $f$ and $g$.

Definition 3.3. Let $A_{0}, A_{1}, \cdots, A_{m-1}$ be nonempty closed subsets of a metric space $(X, d), Y:=\bigcup_{i \in \mathbb{N}_{0}^{m-1}} A_{i}$ and $f, g: Y \rightarrow Y$ be two mappings. If the following conditions are satisfied:

1) $\bigcup_{i \in \mathbb{N}_{0}^{m-1}} A_{i}$ is a co-cyclic representation of $Y$ between $f$ and $g$;

2) there exists $0 \leq \alpha<1$ such that

$$
\begin{gathered}
d(f x, f y) \leq \alpha \max \{d(g x, g y), d(f x, g x), d(f y, g y), \\
d(f x, g x), d(f y, g x)\}
\end{gathered}
$$

whenever $x$ and $y$ are descendants in $Y$, then we say that $f$ and $g$ are $(f, g)$-co-cyclic quasi-contraction.

Remark 3.4. The notions and results in this section can be reduced from the results of the previous section if the mapping $g$ is the identity mapping.

Now, we are ready to give some extensions of the results in Section 2.

Theorem 3.5. Let $A_{0}, A_{1}, \cdots, A_{m-1}$ be nonempty closed subsets of a complete metric space $(X, d)$ and $Y:=\bigcup_{i \in \mathbb{N}_{0}^{m-1}} A_{i}$. Suppose that $f, g: Y \rightarrow Y$ are $(f, g)$ co-cyclic quasi-contraction with $\alpha \in\left[0, \frac{1}{2}\right)$,

$\left.g\right|_{A_{i}}: A_{i} \rightarrow A_{i}$ is a mapping and $g A_{i}$ is closed for all $i \in \mathbb{N}_{0}^{m-1}$. Then $f$ and $g$ have a unique point of coincidence in $\bigcap_{i \in \mathbb{N}_{0}^{m-1}} A_{i}$.

Theorem 3.5 can be proved using the analogous ideas of the proofs in Section 2. However, we prove Theorem 3.5 differently by using the following lemma ([8]):

Lemma 3.6. [8] Let $X$ be a nonempty set and $f: X \rightarrow X$ be a mapping. Then there exists a subset $E \subseteq X$ such that $f(E)=f(X)$ and $f: E \rightarrow X$ is an injection.

Proof of Theorem 3.5. By Lemma 3.2, for each $i \in \mathbb{N}_{0}^{m-1}$, there exists $E_{i} \subseteq A_{i}$ such that $g\left(E_{i}\right)=$ $g\left(A_{i}\right)$ and $g: E_{i} \rightarrow A_{i}$ is an injection. Define $W:=\bigcup_{i \in \mathbb{N}_{0}^{m-1}} g E_{i}$ and a mapping $h: W \rightarrow W$ by $h g x=$ $f x$. It is easy to see that

$$
h\left(g\left(E_{i}\right) \subseteq g\left(E_{i+1(\bmod m)}\right)\right.
$$

for each $i \in \mathbb{N}_{0}^{m-1}$, which further implies that $\bigcup_{i \in \mathbb{N}_{0}^{m-1}} g E_{i}$ is a cyclic representation of $W$ with respect to $h$. More- over, we can write the $(f, g)$-co-cyclic quasi-contraction with $\alpha \in\left[0, \frac{1}{2}\right)$ in terms of a cyclic quasi-contraction as follow:

$$
\begin{aligned}
d(h g x, h g y) \leq & \alpha \max \{d(g x, g y), d(h g x, g x), \\
& d(h g y, g y), d(h g x, g y), d(h g y, g x)\} .
\end{aligned}
$$

Since $W$ is complete, by using Theorem 2.3 , we show that there exists a unique fixed point $g x^{*}$ of $h$ in

$\bigcap_{i \in \mathbb{N}_{0}^{m-1}} g\left(E_{i}\right)$. In fact, this means $g x^{*}=h g x^{*}=f x^{*}=: w$.

Furthermore, we can see that $w$ is also the unique point of coincidence of $f$ and $g$ in $Z:=\bigcap_{i \in \mathbb{N}_{0}^{m-1}} A_{i}$. This completes the proof.

Note that our conditions are not strong enough to show the existence of a common fixed point of two mappings. To guarantee the existence and uniqueness of a common fixed point, we need an additional condition, namely, a weak compatibility, which is defined as follows:

Definition 3.7. [9] Let $X$ be a nonempty set. Two mappings $f, g: X \rightarrow X$ are said to be weakly compatible if they commute at their point of coincidences, i.e., if $x \in X$ is such that $f x=g x$, then $f g x=g f x$.

Theorem 3.8. Suppose that all the conditions in Theorem 3.5 hold. If $f$ and $g$ are weakly compatible, then $f$ and $g$ have a unique common fixed point.

Proof. Since all the conditions in Theorem 3.5 hold, it follows that $f$ and $g$ have a unique point of coincidence $w$ of $f$ and $g$, that is, $w=f x^{*}=g x^{*}$ in $Z:=\bigcap_{i \in \mathbb{N}_{0}^{m-1}} A_{i}$. If $f$ and $g$ are weakly compatible, we have

$f w=f g x^{*}=g f x^{*}=g w$. This means $f w=g w$ is also a point of coincidence of $f$ and $g$. Since the point of coincidence of $f$ and $g$ is unique, we have that $f w=g w=w$, that is, $w$ is a common fixed point between $f$ and $g$.

For the uniqueness of the point $w$, suppose that $f z=g z=z$. Hence $z$ is a point of coincidence of $f$ and $g$. Since the point of coincidence of $f$ and $g$ is unique, we conclude that $z=w$. Thus $f$ and $g$ have a unique common fixed point $w$ in $Z$. This completes the proof.

Theorem 3.9. Let $A_{0}, A_{1}, \cdots, A_{m-1}$ be nonempty closed subsets of a complete ultrametric space $(X, d)$ and $Y:=\bigcup_{i \in \mathbb{N}_{0}^{m-1}} A_{i}$. Suppose that $f, g: Y \rightarrow Y$ are $(f, g)$ co-cyclic quasi-contraction, $\left.g\right|_{A_{i}}: A_{i} \rightarrow A_{i}$ is a mapping and $g A_{i}$ is closed for all $i \in \mathbb{N}_{0}^{m-1}$. Then $f$ and $g$ have a unique point of coincidence in $\bigcap_{i \in \mathbb{N}_{0}^{m-1}} A_{i}$. Moreover, if $f$ and $g$ are weakly compatible, then $f$ and $g$ have a unique common fixed point.

The proof of this theorem can be completed using the proof of Theorems 2.6, 3.5 and 3.8 and so we omit here.

Corollary 3.10. Let $A_{0}, A_{1}, \cdots, A_{m-1}$ be nonempty closed 
subsets of a complete metric space $(X, d)$ and $Y:=\bigcup_{i \in \mathbb{N}_{0}^{m-1}} A_{i}$. Let $f$ and $g$ be two self-mappings on $Y$. Suppose that there exist $\alpha, \beta, \gamma, \delta, \varepsilon \in[0,+\infty)$ such that

$$
\begin{aligned}
d(f x, f y) \leq & \alpha d(g x, g y)+\beta d(f x, g x)+\gamma d(f y, g y) \\
& +\delta d(f x, g x)+\varepsilon d(f y, g x)
\end{aligned}
$$

whenever $x$ and $y$ are descendants in $Y$, where $\alpha+\beta+\gamma+\delta+\varepsilon<\frac{1}{2},\left.g\right|_{A_{i}}: A_{i} \rightarrow A_{i}$ is a mapping and $g\left(A_{i}\right)$ is closed for all $i \in \mathbb{N}_{0}^{m-1}$. Suppose that $\bigcup_{i \in \mathbb{N}_{0}^{m-1}} A_{i}$ is a co-cyclic representation of $Y$ between $f$ and $g$. Then $f$ and $g$ have a unique point of coincidence in $\bigcap_{i \in \mathbb{N}_{0}^{m-1}} A_{i}$. Moreover, if $f$ and $g$ are weakly compatible, then $f$ and $g$ have a unique common fixed point.

Proof. Since $f$ and $g$ satisfy the inequality (3), we can deduce that

$$
\begin{aligned}
d(f x, f y) \leq & (\alpha+\beta+\gamma+\delta+\varepsilon) \max \{d(g x, g y), \\
& d(f x, g x), d(f y, g y), d(f x, g x), d(f y, g x)\}
\end{aligned}
$$

Now, since $(\alpha+\beta+\gamma+\delta+\varepsilon) \in\left[0, \frac{1}{2}\right)$, applying Theorems 3.5 and 3.8, we obtain the result.

Corollary 3.11. Let $A_{0}, A_{1}, \cdots, A_{m-1}$ be nonempty closed subsets of a complete ultrametric space $(X, d)$ and $(X, d) Y:=\bigcup_{i \in \mathbb{N}_{0}^{m-1}} A_{i}$. Let $f$ and $g$ be two self-mappings on $Y$. Suppose that there exist $\alpha, \beta, \gamma, \delta, \varepsilon \in[0,+\infty)$ such that

$$
\begin{gathered}
d(f x, f y) \leq \alpha d(g x, g y)+\beta d(g x, g y)+\gamma d(f y, g y) \\
x+\delta d(f x, g y)+\varepsilon d f(y, g x)
\end{gathered}
$$

whenever $x$ and $y$ are descendants in $Y$, where $\alpha+\beta+\gamma+\delta+\varepsilon<1,\left.g\right|_{A_{i}}: A_{i} \rightarrow A_{i}$ and $g\left(A_{i}\right)$ is closed for all $i \in \mathbb{N}_{0}^{m-1}$. Suppose that $\bigcup_{i \in \mathbb{N}_{0}^{m-1}} A_{i}$ is a co-cyclic representation of $Y$ between $f$ and $g$. Then $f$ and $g$ have a unique point of coincidence in $\bigcap_{\in \mathbb{N}_{0}^{m-1}} A_{i}$. Moreover, if $f$ and $g$ are weakly compatible, then $f$ and $g$ have a unique common fixed point.

Proof. Since $f$ and $g$ satisfy the inequality (3), it follows that

$$
\begin{aligned}
d(f x, f y) \leq & (\alpha+\beta+\gamma+\delta+\varepsilon) \max \{d(g x, g y), \\
& d(f x, g x), d(f y, g y), d(f x, g y), d(f y, g x)\} .
\end{aligned}
$$

Now, since $(\alpha+\beta+\gamma+\delta+\varepsilon) \in[0,1)$, applying Theorem 3.4, we obtain the result.

If $g$ is the identity mapping in Corollaries 3.5 and 3.6, we have the following:

Corollary 3.12. Let $A_{0}, A_{1}, \cdots, A_{m-1}$ be nonempty closed subsets of a complete metric space $(X, d)$ and $Y:=\bigcup_{i \in \mathbb{N}_{0}^{m-1}} A_{i}$. Let $f$ be a self-mapping on $Y$. Suppose that there exist $\alpha, \beta, \gamma, \delta, \varepsilon \in[0,+\infty)$ such that

$$
\begin{aligned}
d(f x, f y) \leq & \alpha d(x, y)+\beta d(x, f x)+\gamma d(y, f y) \\
& +\delta d(x, f y)+\varepsilon d(y, f x)
\end{aligned}
$$

whenever $x$ and $y$ are descendants in $Y$, where $\alpha+\beta+\gamma+\delta+\varepsilon<\frac{1}{2}$. Suppose that $\bigcup_{i \in \mathbb{N}_{0}^{m-1}} A_{i}$ is a cyclic representation of $Y$ with respect to $f$. Then $f$ has a unique fixed point in $\bigcap_{i \in \mathbb{N}^{m-1}} A_{i}$.

Corollary 3.13. Let $A_{0}, A_{1}, \cdots, A_{m-1}$ be nonempty closed subsets of a complete ultrametric space $(X, d)$ and $Y:=\bigcup_{i \in \mathbb{N}_{0}^{m-1}} A_{i}$. Let $f$ be a self-mapping on $Y$. Suppose that there exist $\alpha, \beta, \gamma, \delta, \varepsilon \in[0,+\infty)$ such that

$$
\begin{aligned}
d(f x, f y) \leq & \alpha d(x, y)+\beta d(x, f x)+\gamma d(y, f y) \\
& +\delta d(x, f y)+\varepsilon d(y, f x)
\end{aligned}
$$

whenever $x$ and $y$ are descendants in $Y$, where $\alpha+\beta+\gamma+\delta+\varepsilon<1$. Suppose that $\bigcup_{i \in \mathbb{N}_{0}^{m-1}} A_{i}$ is a cyclic representation of $Y$ with respect to $f$. Then $f$ has a unique fixed point in $\bigcap_{i \in \mathbb{N}_{0}^{m-1}} A_{i}$.

Remark 3.14. Notice that Corollary 3.12 also generalizes the condition $\alpha+\beta+\gamma+2 \delta<\frac{1}{2}$ of Theorem 1.2.

\section{Conclusion}

For the single-mapping case, the existence and uniqueness of a fixed points for a quasi-contraction in cyclic sense is proved with a restriction that the contraction constant have to be less than $\frac{1}{2}$. We further showed that if $X$ is an ultrametric space, such a restriction may be dropped. Further, with the notion of a co-cyclic representation, we point out that the two-mapping case may be extended from our results proved earlier.

\section{Acknowledgements}

The authors were supported by the Higher Education Research Promotion and National Research University Project of Thailand, Office of the Higher Education Commission (NRU-CSEC No.55000613). The second author was supported by the Basic Science Research Program through the National Research Foundation of Korea (NRF) funded by the Ministry of Education, Science, and Technology (Grant no. 2011-0021821). The Third author would like to thank the Research Professional Development Project Under the Science Achievement Scholarship of Thailand (SAST). 


\section{REFERENCES}

[1] S. Banach, "Sur les Opérations Dans les Ensembles Abstraits Et Leur Applications Aux Équations Intégrales," Fundamenta Mathematicae, Vol. 3, 1922, p. 160.

[2] Lj. Ćirić, "Generalized Contractions and Fixed-Point Theorems," Publications de l'Institut Mathématique, Vol. 12, No. 26, 1971, pp. 19-26.

[3] Lj. B. Ćirić, "A Generalization of Banach's Contraction Principle," Proceedings of the American Mathematical Society, Vol. 45, No. 2, 1974, pp. 267-273. doi: $10.2307 / 2040075$

[4] I. A. Rus, "Cyclic Representations and Fixed Points," Ann. T. Popoviciu Seminar Funct. Eq. Approx. Convexity, Vol. 3, 2005, pp. 171-178.

[5] M. Păcurar and I. A. Rus, "Fixed Point Theory for Cyclic $\phi$-Contractions," Nonlinear Analysis: Theory, Methods \& Applications, Vol. 72, No. 3-4, 2010, pp. 1181-1187. doi:10.1016/j.na.2009.08.002
[6] E. Karapinar, "Fixed Point Theory for Cyclic Weak $\phi$ Contraction," Applied Mathematics Letters, Vol. 24, No. 6, 2011, pp. 822-825. doi:10.1016/j.aml.2010.12.016

[7] W. Sintunavarat and P. Kumam, "Common Fixed Point Theorem for Cyclic Generalized Multi-Valued Contraction Mappings," Applied Mathematics Letters, Vol. 25, No. 11, 2012, pp. 1849-1855. doi:10.1016/j.aml.2012.02.045

[8] R. H. Haghi, Sh. Rezapour and N. Shahzad, "Some Fixed Point Generalizations Are Not Real Generalizations," Nonlinear Analysis: Theory, Methods \& Applications, Vol. 74, No. 5, 2011, pp. 1799-1803. doi:10.1016/j.na.2010.10.052

[9] G. Junck and B. E. Rhoades, "Fixed Point for Set Valued Functions without Continuity," Indian Journal of Pure and Applied Mathematics, Vol. 29, No. 3, 1998, pp. 227 238. 